\title{
Comparison of Two Kinds of CFA Algorithms in Iris Identification Hongqi $\mathrm{Li}^{1, \mathrm{a}}$, Yan Deng ${ }^{1, \mathrm{~b}}$ and Wei Su${ }^{1, \mathrm{c}^{*}}$ \\ ${ }^{1}$ Institute of mechanical and electrical engineering of Hankou College, Hubei Province, China a359161557@qq.com, b119076870 qq.com, csuw0801@126.com \\ *The corresponding author
}

Keywords: Iris recognition; CFA algorithm; CCD; SCEE algorithm

\begin{abstract}
In an iris recognition system, the iris image quality of CCD signal acquisition is the key factor of system identification. Two kinds of CFA algorithms in iris recognition system image processing module are compared in this paper. Compared with SC interpolation algorithm, SCEE algorithm can improve the image transformation in the gradient direction. The superiority of SCEE algorithm is verified by matlab simulation.
\end{abstract}

\section{Introduction}

Biological recognition technology could identify a person (whom he or she is) or validate the person (whether he or she is the claimed one) using human peculiar physiological or behavioral characteristics (like face, fingerprint, palmprint, palm vein, iris and retina gait, etc.). Different from traditional password keys which could be easy to forget or lost, those special characteristics have high security, high practicality, and high accuracy, which could solve almost all the problems referred above about traditional ways, so it has been widely concerned in security fields such as airport security as well as bank security, among them, the iris identification is proved to have the highest accuracy[1], and CFA ( Color Filter Array) plays an important role in iris recognition systems. In this paper, we focused on studying the difference of the theory in two CFA algorithms, and verifying the performance through different experiments.

\section{Theory of the Iris Identification}

Iris recognition is divided into two different processes: registration and certification/recognition. The previous steps of both are the same: after collecting the iris image, localization and normalization are made to them (some need image enhancement and filtering for pretreatment), which could help the ring shape of iris into a unified rectangular block, then using image enhancement and algorithm to extract certain features to obtain the iris feature (iris code). The differences lay in: the obtained code will be stored in the database as an object of the template in registration process, whereas the obtained code needs comparison beforehead with the template in the datebase to check out whether this code is the claimed one in "certification/identification process".

The whole iris recognition system includes three parts; they are iris image acquisition module part, image processing module part and intelligent recognition module part respectively, just as shown in Fig. 1. The true color image for restoring CCD acquisition in image processing module part involves two crucial steps: introducing CFA to form RGB mosaic image in image acquisition process, and restoring true color image through CFA interpolation image correction[2,3], each of the pixel color component of the filtered imaged from CFA consists only single color, by local amplifying the image, we can find that the image is a mono-color of mosaic image, so the other two primary colors are added to each pixel to obtain a full color image, this is called CFA interpolation, also called color interpolation as well. 


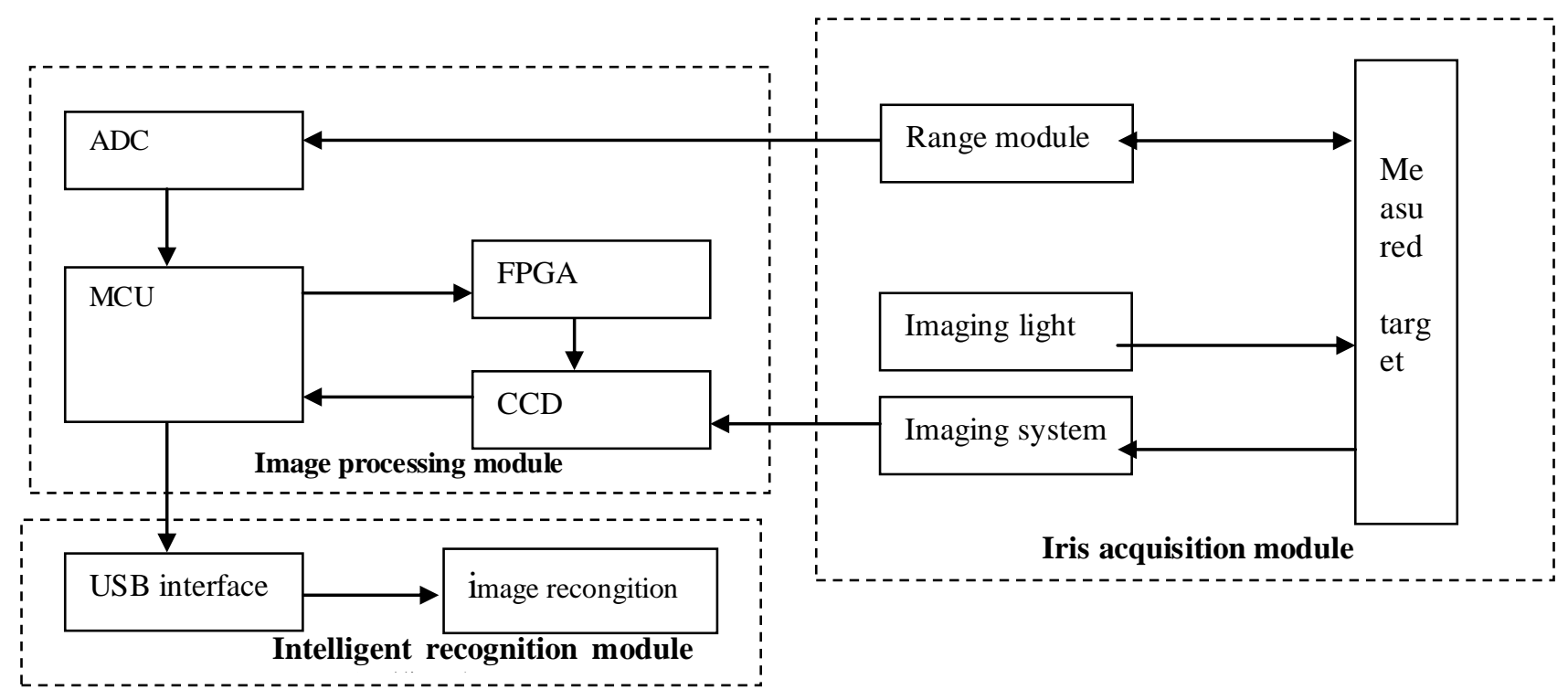

Figure 1. system structure

\section{Theory Analysis for 2 Kinds of CFAAlgorithms}

In CFA interpolation, the interpolated pixel information could be evaluated through calculating the neighboring pixels information, which can inevitably exist a bias compared with the original image, image edge area as well as where color changes obviously in particular, and a significant distortion may appear[4-6].

In common interpolation algorithm, the existing channel $\mathrm{G}$ information is used to restore the lost $G$ values, while, there is a strong relevance among channel $R$, channel $G$ and channel $B$, which means that, the interpolation in channel $\mathrm{G}$ can also use the information from channel $\mathrm{R}$ as well as channel $\mathrm{B}$. to achieve this, the previous researchers came up to an image module based on the relevance of channel $R$, channel $G$ and channel $B$, which is called Signal correlation algorithm( "SC"). this SC algorithm is based on colorimetric constant interpolation algorithm, and is assumed that, the surrounding interpolated pixels the $\mathrm{R}$ and $\mathrm{B}$ have a relevance to $\mathrm{G}$. Based on this module, those researchers defined that, $\mathrm{G}$ minus $\mathrm{R}$ equals to $\mathrm{Kr}, \mathrm{G}$ minus $\mathrm{B}$ equals to $\mathrm{Kb}$, just as shown below:

$$
\left\{\begin{array}{l}
\mathrm{K}_{r}=G-R \\
\mathrm{~K}_{b}=G-B
\end{array}\right.
$$

For real image, the comparison of $\mathrm{Kr}$ and $\mathrm{Kb}$ is very flat in a small area, which could be a perfect match for interpolation, this algorithm is easier to conduct in FPGA $[7,8]$.

Along with the enhancement of CCD, the ISP (image signal processor) which is based on RAW format becomes more and more popular, combined with its strong parallel operating capability, more and more algorithms are made in FPGA[9,10]. Whereas in ISP algorithm, interpolating to the RAW data is one of the most important module. There presents a new interpolation algorithm based on SC algorithm, namely signal correlation edge enhance (SCEE). The enhancement is that: it introduces gradient information of decision to complete the lack of color correlation in gradient direction in SC. since we human are more sensitive to light and green color makes the greatest contribution, there has little change in color when $\mathrm{SC}$ algorithm is transformed to $\mathrm{Kb}$ and $\mathrm{Kr}$ space, so the interpolation error there reduced greatly. But $\mathrm{Kb}$ and $\mathrm{Kr}$ are not completely the same color, there is still some difference in the texture, so vertical information decision is introduced when calculating the brightness, and the formula is shown as follows:

$$
G(i, j)=I(i, j)+f(K(i, j))
$$

$\mathrm{I}(\mathrm{i}, \mathrm{j})$ is the RAW value for $\mathrm{R}$ or $\mathrm{B}$, the definition of function $\mathrm{f}(\mathrm{K}(\mathrm{i}, \mathrm{j})$ is shown in $\mathrm{Eq} .3, \Delta \mathrm{h}$ is 
horizontal gradient operator, $\Delta \mathrm{v}$ is vertical gradient operator.

$$
f_{S C E E}(K(i, j))=\left\{\begin{array}{c}
\frac{K(i-1, j)+K(i+1, j)}{2} \quad \text { if } \quad \Delta v>\Delta h \\
\frac{K(i, j-1)+K(i, j+1)}{2} \quad \text { if } \Delta v<\Delta h \\
\frac{K(i-1, j)+K(i+1, j)+K(i, j-1)+K(i, j+1)}{4} \quad \text { else }
\end{array}\right.
$$

SCEE has both space conversion compared with SC algorithm, it also introduces horizontal and vertical information decision. When calculating $B$ in $R$, as well as $R$ in $B$, the function $f(K(i, j))$ can also be calculated using Eq. 3 .

$$
R B(i, j)=G(i, j)-f_{S C E E}(K(i, j))
$$

\section{Matlab Interpolation Simulation}

Through algorithm simulation in matlab, the result shows the algorithm in Matlab, Fig. 2(a) is simulating the original CFA format image, Fig. 2 (b) is obtained afterward SCEE interpolation, Fig. 2 (c) is an original standard RGB image. We can see in Fig. 2 that the interpolation result is consist with the original image, while amplifying the (b)and (c) through matlab, there still exist some problems.

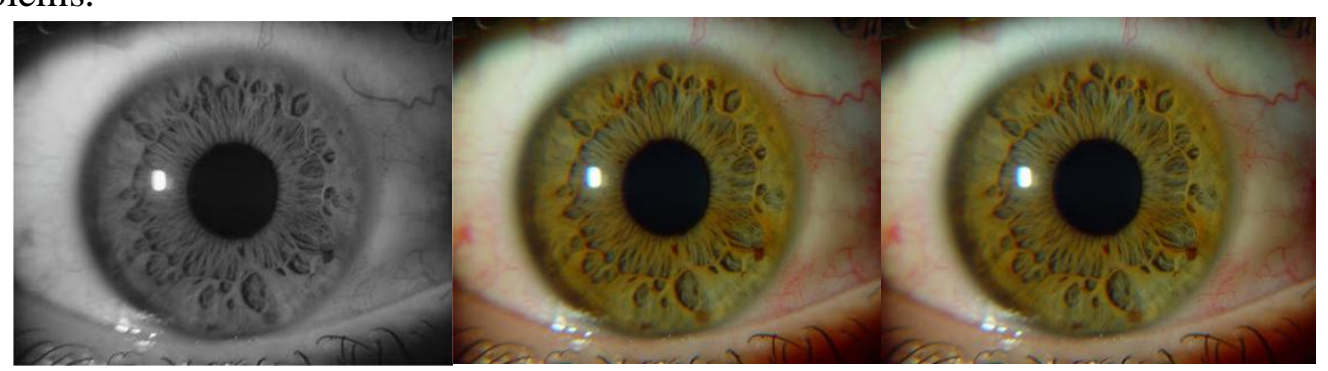

(a)

(b)

(c)

Figure 2. simulation image for interpolation

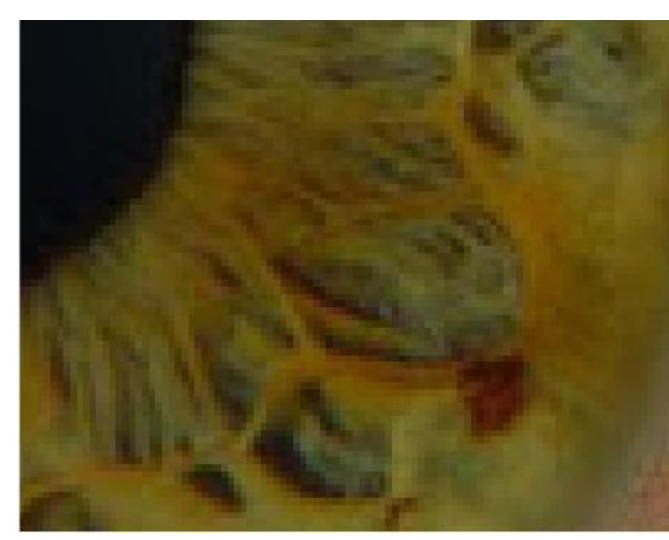

(a)

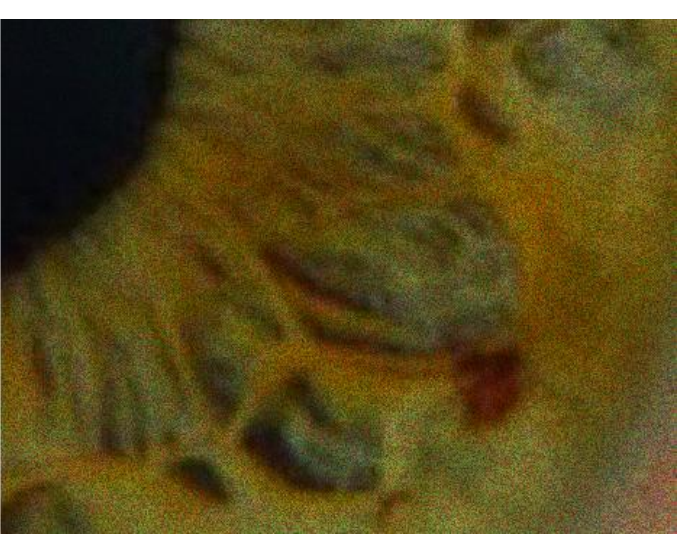

(b)

Figure 3. details enlarged drawing

Fig. 3(a) is a raw image, 3(b) is obtained after stripping out from RAW date and after simulating difference. Compared with 3(a) and 3(b) we can easily discover that, there exists errors not only in color, some edge area also appears a distortion to some degree.

In order to evaluate the performance of interpolation algorithm, the signal-to-noise ration index is introduced: 


$$
P S N R=10 \log _{10}\left(255^{2} / \sum_{i=1}^{n} \sum_{j=1}^{m}\left(I_{i j}-I_{i j}^{\prime}\right)^{2}\right)
$$

The "I" is source image, which generates RAW image through simulation, I' is image after interpolation. The method of generating a RAW image is: extract the source image from each pixel's RBG components, then each pixel distributes information based on three primary colors according to BGR sequence, the grayscale image, namely RAW image is formed. Each algorithm can make a simulation comparison in this matlab with this method, and the signal-to-noise ratio is arranged according to RBG trichromatic order. The SC algorithm coordination is 29.86、33.15、 30.03 respectively; The SCEE algorithm coordination is 31.96、34.68、32.30 respectively. So from the view of signal-to-noise ratio, SCEE algorithm overweighs SC algorithm.

\section{Conclusion}

This paper studied the CFA algorithm in iris recognition system so as to enhance the validity as well as the quality of iris image. Although the SC algorithm successfully makes a color-to-space conversion, it ignored the fact that $\mathrm{Kb}$ and $\mathrm{Kr}$ are not completely flat data, they also exist texture in space. So, this paper came up with the SCEE algorithm to enhance the current SC interpolation algorithm which can't transform images in the direction of all gradients, simulation verification is also made through matlab to test its priority. Through FPGA design to SCEE algorithm, the iris image acquisition and processing can be achieved based on it.

\section{Acknowledgements}

This study was supported by "Research of intelligent recognition based on iris recognition" (Project No. B2014122) and "LED LCOS light source optical engine efficiency research" (Project No. B2016321) funding, which are Education Department of Hubei Province scientific research plan project.

\section{References}

[1] C.T.Sun: Research on Algorithms of Color Image Based Iris Recognition (Ph.D., Jilin University, China 2009).

[2] Y.H.Dai: Research on Color Interpolation Method of Digital Image (Ph.D., University of Electronic Science and technology, China 2013).

[3] Adams, J. E., Hamilton, J. F: Design of practical color filter array interpolation algorithms for digital cameras ( Bellingham, USA, 1997 ). vol.3028, p.117-25).

[4] X.L.Zhang, Z. Fang and X.P. Zhang: Journal of Applied Science, vol.33(2015),p87-94.

[5] S.Peng,Y.Y. Peng and C.Y. Xiao: Sensors and micro systems, vol.34(2015),p141-144.

[6] J.Gao,X.Y.Li and X.F. Ren: Information communication, vol.13(2015),p1-3.

[7] C.J.Chen: Design and implementation of Bayer image color interpolation system based on FPGA (Ph.D., University of Chinese Academy of Sciences, China 2010).

[8] P.Xia,S.G. Zhang and N.Lei.: Journal of Computational and Applied Mathematics, vol.23 (2011).

[9] Huaping Ma: Research And FPGA Realization of Color Interpolation Algorithms in Digital Cameras (Ph.D., University of Electronic Science and technology, China 2013).

[10] Jianzhuang Wang: Study on the Algorithms of High-speed Image Processing Based on FPGA and System Implementation (Ph.D., Huazhong University of Science and Technology, China 2011). 